\title{
Can "Touch" Get Annoying?
}

\author{
Jens Gerken, Hans-Christian Jetter, Toni Schmidt, and Harald Reiterer \\ University of Konstanz, HCI Group \\ Konstanz, Germany \\ firstname.lastname@uni-konstanz.de
}

\begin{abstract}
While touch interaction with tabletops is now widely accepted as a very natural and intuitive form of input, only little research has been carried out to understand whether and how it might interfere with our natural ways of gestural communication. This poster presents a study that aims at understanding the importance of touching physical and virtual artifacts during discussion or collaboration around a table. Furthermore, it focuses on how users compensate for conflicts between non-interactivity and interactivity created by unintended touch interaction when using a multi-touch enabled tabletop. In our study, we asked participants to explain illustrations of technical or physical mechanisms, such as the workings of an airplane wing. We observed whether and how they used gestures to do so on a touch sensitive Microsoft Surface tabletop and on a sheet of paper. Our results suggest that touching is an essential part of such an activity and that the compensation strategies people adapt to avoid conflicts may reduce precision of communication and increase the physical strain on the user.
\end{abstract}

ACM Classification: H5.2 [Information interfaces and presentation]: User Interfaces. - Graphical user interfaces.

General terms: Design, Human Factors

Keywords: Touch, tabletop, gestures, touch-sensitivity.

\section{INTRODUCTION}

Touch-enabled surfaces have now become ubiquitous in tabletop computers, tablet PCs, or smart phones. While much research has been done on how to make surfaces interactive, it is rarely discussed under which circumstances this interactivity becomes disruptive. As has been discussed by O'Hara, the role of non-interactivity can be very important in typical public settings with tabletops where unintended interaction can constrain non-interactive intentions [3]. Temporary non-interactivity is also discussed by Jacob et al. [1] in connection with the Apple iPhone: This device automatically turns off its touch screen as soon as it is moved towards the ear in an upright position to avoid unintended touches that could lead to unintentionally aborting the call. Our goal is to better understand when touch input creates such conflicts between the interactivity of a surface and our familiar ways of pointing or gesturing. By this, we hope to lay the foundation of a conceptual framework that could help designers to make touch smarter and more context-aware in future.

\section{EXPERIMENT}

Our observational study was inspired by the work of O'Hara and has been designed to reveal more detailed insight about the relation between intended and unintended

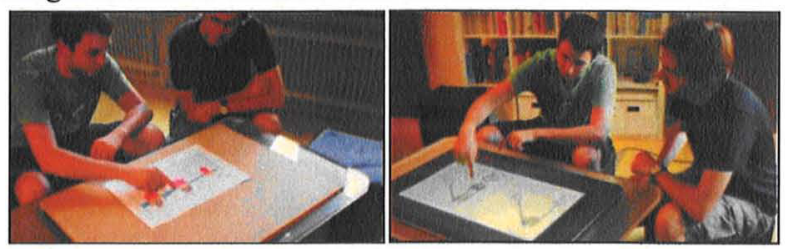

Figure 1: left - paper condition, right - Surface condition

touch input during group discussion or collaboration around a tabletop. While observing such situations in the real world, we found anecdotal evidence for frequent unintended touch inputs causing lots of disruptions: Frequently users made unintentional touch inputs with their fingers and by this unintentionally changed the state of the user interface. However, their actual intention was only to point at an object for better communicating with others, but not to actually manipulate or interact with it. To further investigate this, we observed student participants from various subjects $(n=11,10$ female, age 19-26) during the task of explaining a complex artifact to the experimenter (fig. 1). As artifacts we chose 12 illustrations explaining the workings of technical and physical mechanisms from a children's book [2] (fig. 2). Each artifact consisted of an illustration and a short text. Participants were given some time to read the text and then were asked to explain the illustration to the experimenter. To do this, they had to explain and point towards distinct objects in the illustration, very similar to the real life situations in which we had observed unintended touches.

\section{Conditions}

Each participant alternated for each of the 12 artifacts between two conditions, thus explaining half of the artifacts on the Surface (fig. 1, right) and the other half on paper (fig. 1, left). The purpose of this alternation was to simulate a realistic environment in which both touch-sensitive 
surfaces (e.g. tabletops, tablet PCs) and touch-insensitive surfaces (e.g. stylus-only tablet PCs or paper) are used during collaboration. The paper condition also served us as some kind of "reset" to make users return subconsciously to their natural ways of touching and pointing. Thereby, we avoided that they quickly adopted an artificial Surfaceinduced "no touch" pointing style. Since we were also interested in the strategies, that users would employ to compensate for undesired touches, every touch on the Surface lead to a non-destructive yet annoying zoom-out animation. This rendered the details of the illustration too small to read. A subsequent tap returned to the original display size again.
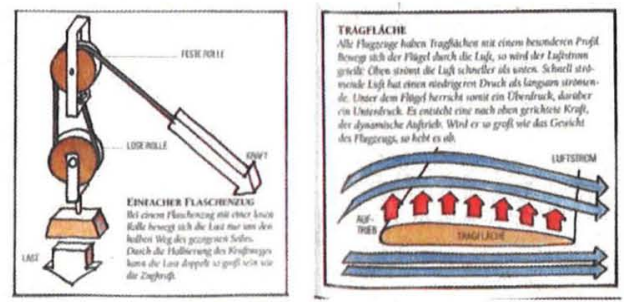

Figure 2: Example tasks pulley (left) and airplane wing (right)

\section{Findings}

We observed three characteristic patterns in participants' behavior that have been reoccurring during the study:

Touch is essential for human communication - During the paper condition, $50 \%$ of all gestures included physical touches for pointing at parts of the artifact. Touching while pointing is not something that just happens accidentally from time to time but is an essential part of the explanation process in a natural paper-based environment. When looking at the Surface condition, we can confirm this interpretation as seven out of our eleven participants tried to carry over this behavior and did touch the display during their explanations. Being confronted with the resulting unintended zoom-out animation, all but one then successfully applied compensation strategies and reduced touching to a minimum (avg. $8 \%$ of gestures). However, as we discuss below, these strategies might have negative implications on the process of explanation.

Affordance vs. touch-phobia? Interestingly, four out of eleven participants did not touch the tabletop at all, although all of them heavily relied on touch gestures during the paper condition and had not yet experienced an unintended zoom-out. These participants mentioned afterwards that they avoided touching from the start to avoid "changing anything". Furthermore, during the very first "Surface" task (the pulley in fig 2.) only one participant touched the display at all, hinting at initial "notouch" strategies or some kind of "touch-phobia" among the participants. However, in the later course of the study, six participants were not able to keep up this strategy and issued unintended touch input later.
Compensation strategies - All participants (P1-P11) adopted compensation strategies and showed individual differences while doing so in order to avoid touching the Surface. These strategies became particularly obvious during simulation gestures that were used by participants to introduce imaginary dynamic movements into the artifact for illustrating changes over time, e.g. the flow of air:

Hovering - Instead of touching the Surface, some left their fingers hovering at a very small height above the display. P2 applied this strategy throughout all tasks and only once touched the Surface. While this strategy enables users to still point similar to the paper condition, we consider it as potentially harmful: it imposes more physical strain on the user and will demand more attention.

Up in the air - Another strategy was to relocate the simulation gestures well above the Surface and to use the air space above it. This happened nearly twice as often during the Surface condition compared to the paper condition. While reducing physical strain, it makes it more difficult to follow such gestures, as they cannot be easily assigned to the referred parts of the artifact below, thereby reducing the preciseness and fluency of the explanation.

No pointing - As already mentioned, four users simply did not use any pointing gestures at all to avoid touching the Surface. P8 is especially interesting here, as she relied on touch during $90 \%$ of her paper gestures (the highest value) but still avoided any single contact with the Surface display.

Repeated failure - P5 entirely failed at adapting to touchsensitivity. She kept touching the tabletop unintentionally during the whole experiment, so $29 \%$ of all her gestures were unintended touch input. By the end of the study she felt angry about her inability to avoid touching despite being aware of her touches' consequences.

\section{CONCLUSION}

Touch-sensitive interactive surfaces can interfere with our familiar gestures for pointing and explanations during discussion and collaboration. Touch-enabled devices can lead to "touch-phobia" and the reduction or complete avoidance of pointing, which will most likely lead to less efficient and fluent communication. Typical compensation strategies might also increase physical strain and the need for users' attention. It is therefore a worthwhile undertaking to study such interferences further to make "touch" a smarter and more context-aware modality in future.

\section{REFERENCES}

1. Jacob, R. J., et al. 2008. Reality-based interaction: a framework for post-WIMP interfaces. In Proc. CHI 2008, ACM/SIGCHI, NY, 2008, pp. 201-210.

2. Macaulay, D. The way things work ( $2^{\text {nd }}$ edition). Dorling Kindersley Publishers, London, UK, 2004.

3. O'Hara, K. Interactivity and non-interactivity on tabletops. In Proc. CHI'10, ACM/SIGCHI, NY, USA, 2010, pp. 2611-2614. 